Active and Passive Elec. Comp., June 2004, Vol. 27, pp. 107-110

\title{
A LOW-PASS FILTER OF WIDE STOPBAND WITH A NOVEL MULTILAYER FRACTAL PHOTONIC BANDGAP STRUCTURE
}

\author{
HAI-WEN LIU ${ }^{\mathrm{a}, \mathrm{b}, *}$, XIAO-WEI SUN ${ }^{\mathrm{b}}$ and ZHENG-FAN LI ${ }^{\mathrm{a}}$

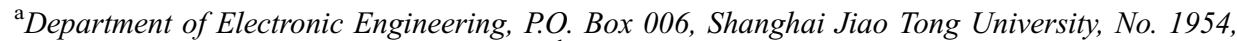 \\ Huashan Rd., Shanghai 200030, China; 'י Shanghai Institute of Microsystem and Information \\ Technology, Chinese Academy of Sciences, Shanghai 200050, China
}

(Received 14 February 2003; In final form 21 March 2003)

\begin{abstract}
A novel, multilayer fractal PBG is presented here as the substrate for a microstrip line and the resulting configuration builds a low-pass filter of wide stopband. Experimental results in comparison with the corresponding two filters with monolayer PBG show that the proposed filter drastically enhances the width of the stopband.
\end{abstract}

Keywords: Photonic bandgap; Low-pass filter; Fractal; Multilayer structure

\section{INTRODUCTION}

Filtering of undesired frequencies in microstrip designs can be implemented with shunt stubs or stepped-impedance lines, but these techniques provide typically narrow-band and a spurious passband in stopband and occupies valuable circuit layout area. Photonic bandgap (PBG) structures have been considered as an alternate to solve this problem in microwave and millimeter-wave circuit applications recently [1-8]. (PBG structures are periodic structures which exhibit a bandgap within a certain band of electromagnetic propagation is prohibited $[9,10])$. But most of the PBG structures show a periodic frequency stopband of which the higher-order stopband is termed harmonic. In order to achieve wide stopband characteristics, several efforts have been proposed using serial or parallel PBG structures to meet this goal, respectively $[11,12]$. But this required large size and had a limitation in microstrip circuits applications in compact sizes. In this letter, a low-pass filter (LPF) of wide stopband with a novel multilayer and fractal photonic bandgap structure is introduced. Moreover, the comparison of an LPF with the conventional PBG structure, an LPF with one-order Sierpinski carpet PBG structure and the proposed LPF is shown. The experimental results of the three LPFs are presented. At the same time, the results show that the transmission characteristic of the proposed LPF is better than the other two.

* Corresponding author. Tel.: (86-21) 62933001; E-mail: haiwen_liu@sjtu.edu.cn 


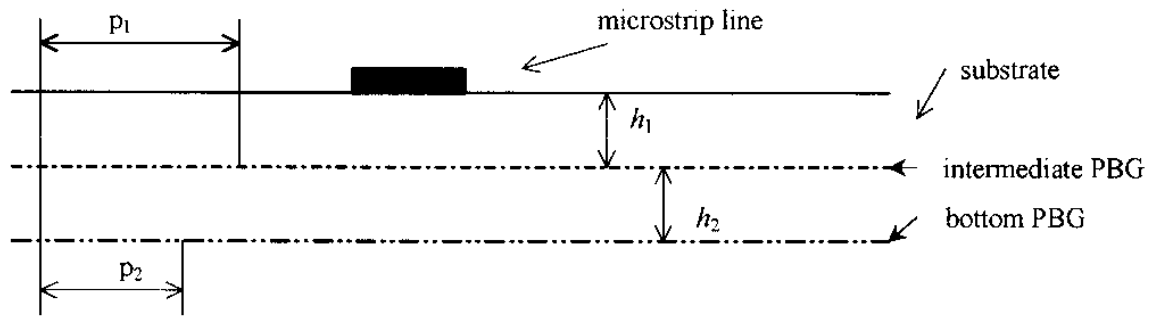

FIGURE 1 Schematic view of the cross-section of the LPF structure.

\section{DESIGN OF THE PROPOSED LPF}

The proposed LPF with multilayer fractal PBG structures is described in Figure 1. It is a microstrip structure in which the ground plate has been replaced by a two-level fractal PBG plate arrangement, and differentiates from the monolayer PBG structure by the addition of the intermediate PBG plate in the substrate. The patterns of the two fractal PBGs are shown in Figure 2. The intermediate fractal PBG structure is made of eight three-order Sierpinski gaskets with a period of $p_{1}=38.2 \mathrm{~mm}$ and the bottom fractal PBG structure is made of seven one-order Sierpinski carpets (in fact, the proposed Sierpinski carpet is not a totally fractal shape, strictly speaking [13]) with a period of $p_{2}=22.6 \mathrm{~mm}$. The bottom PBG is surrounded by a metallic region connected to the ground while the intermediate PBG is grounded by holes. The Teflon-based substrate has a dielectric constant of 2.22 with a thickness of $h_{1}=h_{2}=0.254 \mathrm{~mm}$. A $50-\Omega$ microstrip transmission line on the top plate has a width of $1.77 \mathrm{~mm}$, corresponding to a gap center frequency of $5 \mathrm{GHz}$. The photograph of the structure is shown in Figure 2.

\section{EXPERIMENTAL RESULTS}

Three LPFs with different PBG structures are designed, one LPF having a monolayer PBG structure with typical square holes etched in the ground plane (LPF1), and one LPF having a
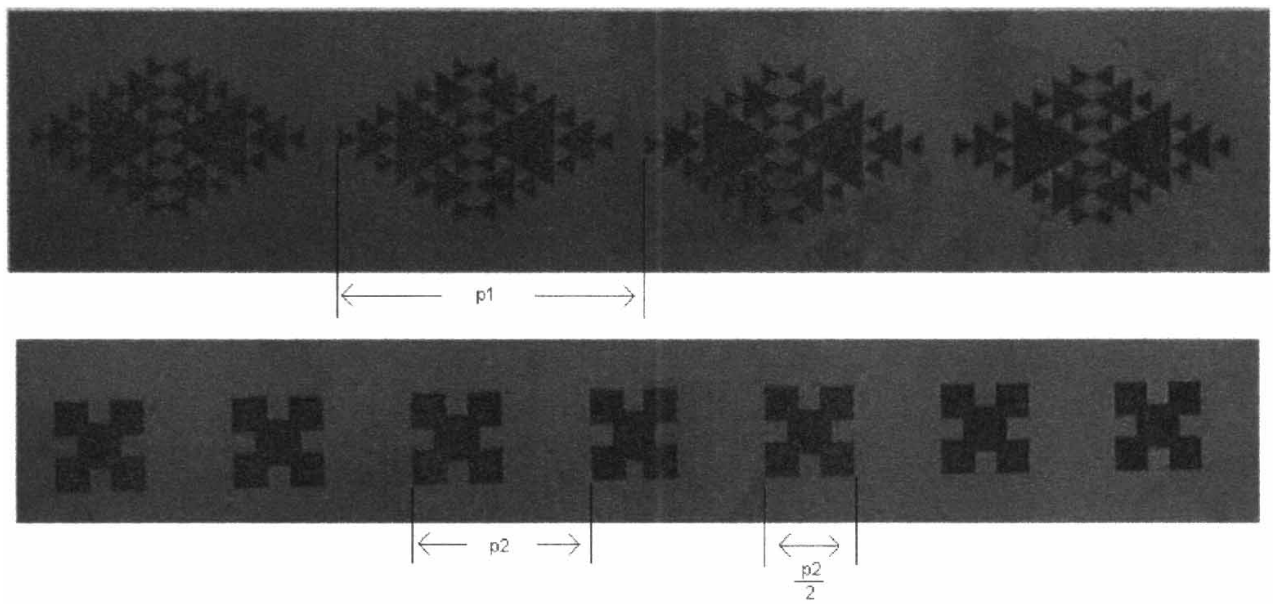

FIGURE 2 Photos of the proposed multilayer fractal PBG structures. The upper is the intermediate PBG, the lower is the bottom PBG. 


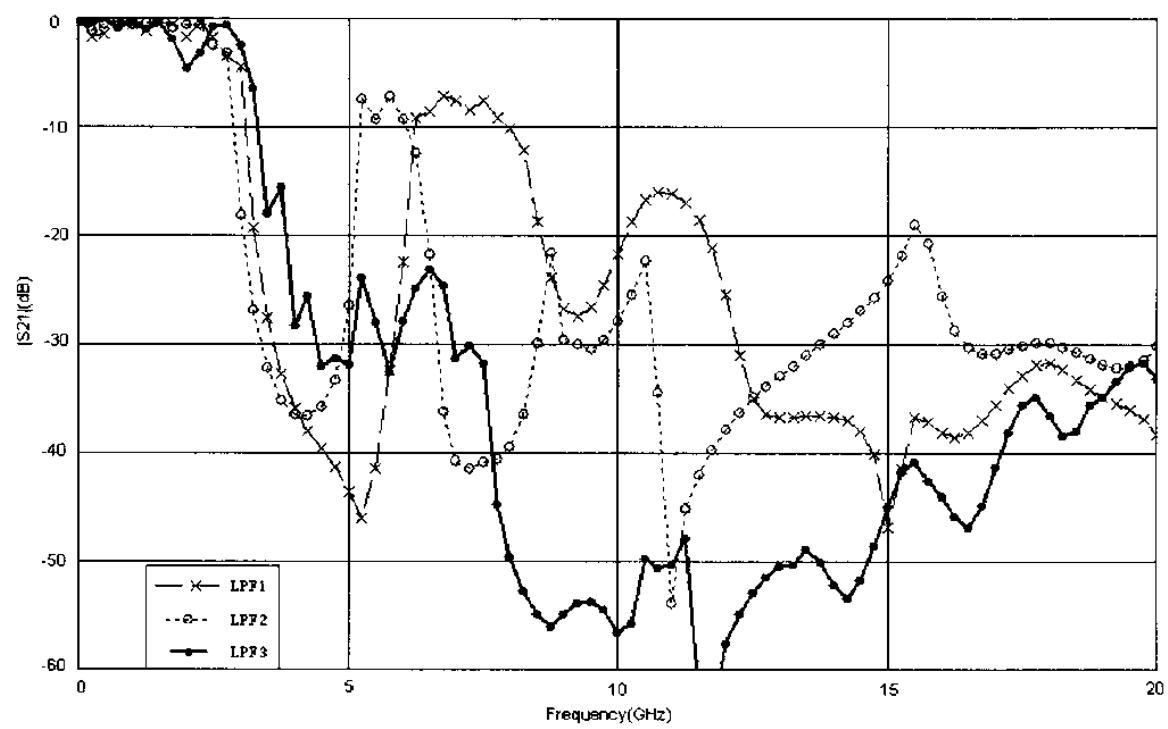

FIGURE 3 Measured results of the transmission characteristics of the three LPFs.

monolayer PBG structure with the proposed one-order Sierpinski carpets etched in the ground plane (LPF2), and the proposed one (LPF3) is described in Figure 1. The experimental results of the transmission characteristics of the three LPFs are shown in Figure 3. In the 3-20 GHz wide stopband seen in Figure 3, the performance of the proposed PBG structure is better than the performance of the other two, and the transmission coefficients of the proposed one are below $-20 \mathrm{~dB}$ while the other two have a transmission peak more than $-10 \mathrm{~dB}$ at about $7 \mathrm{GHz}$. The measurements were performed with a network analyzer (HP $8722 \mathrm{D}$ ) over the frequency range $50 \mathrm{MHz}-40 \mathrm{GHz}$.

\section{CONCLUSIONS}

An LPF with the novel multilayer fractal PBG structures is proposed, realized and tested. In the specific design, the multilayer PBGs include two fractal structures with different patterns. Experimental results in comparison with the corresponding two filters with monolayer PBG show that the proposed filter drastically enhances the width of the stopband.

\section{References}

[1] Laso, M. G., et al. (2000). Novel wideband photonic bandgap structure. Microwave and Optical Tech. Lett., 24, 357-360.

[2] Radisic, V., et al. (1998). Novel 2-D photonic bandgap structure for microstrip lines. IEEE Microwave and Guided Wave Letters, 8, 69-71.

[3] Yang, F. R., et al. (1999). A novel uniplanar compact PBG structure for filter and mixer application. IEEE MTT-S, 47, 1509-1514.

[4] Coccioli, R., et al. (1999). Aperture-coupled patch antenna on UC-PBG substrate. IEEE Trans. MTT, 47, 2123-2130.

[5] Caloz, C. and Itoh, T. (2002). Multilayer and anisotropic planar compact PBG structure for microstrip application. IEEE Trans. MTT, 50, 2206-2208.

[6] Radisic, V., et al. (1998). Broad-band power amplifier using dielectric photonic bandgap structure. IEEE Microwave and Guided Wave Letters, 8, 13-14. 
[7] Rumsey, I., et al. (1998). Photonic bandgap structure used as filters in microstrip circuits. IEEE Microwave and Guided Wave Letters, 8, 336-339.

[8] Laso, M. A. G., et al. (2000). Multiple-frequency-tuned photonic bandgap structure. IEEE Microwave and Guided Wave Letters, 10, 220-222.

[9] Yablonovitch, E. (1987). Inhibited spontaneous emission in solid-state physics and electronics. Phys. Rev. Lett., 58, 2059-2062.

[10] John, S. (1987). Strong localization of photo in certain disordered dielectric superlattices. Phys. Rev. Lett., 58, 2486-2489.

[11] Rumsey, I., et al. (1998). Photonic bandgap structure used as filters in microstrip circuits. IEEE Microwave and Guided Wave Letters, 8, 336-338.

[12] Kim, T. and Seo, C. (2000). A novel photonic bandgap structure for low-pass filter of wide stopband. IEEE Microwave and Guided Wave Letters, 10, 13-15.

[13] Fu, Y. Q., et al. (2002). A novel fractal microstrip PBG structure. Microwave and Optical Tech. Letters, 32, $136-138$. 

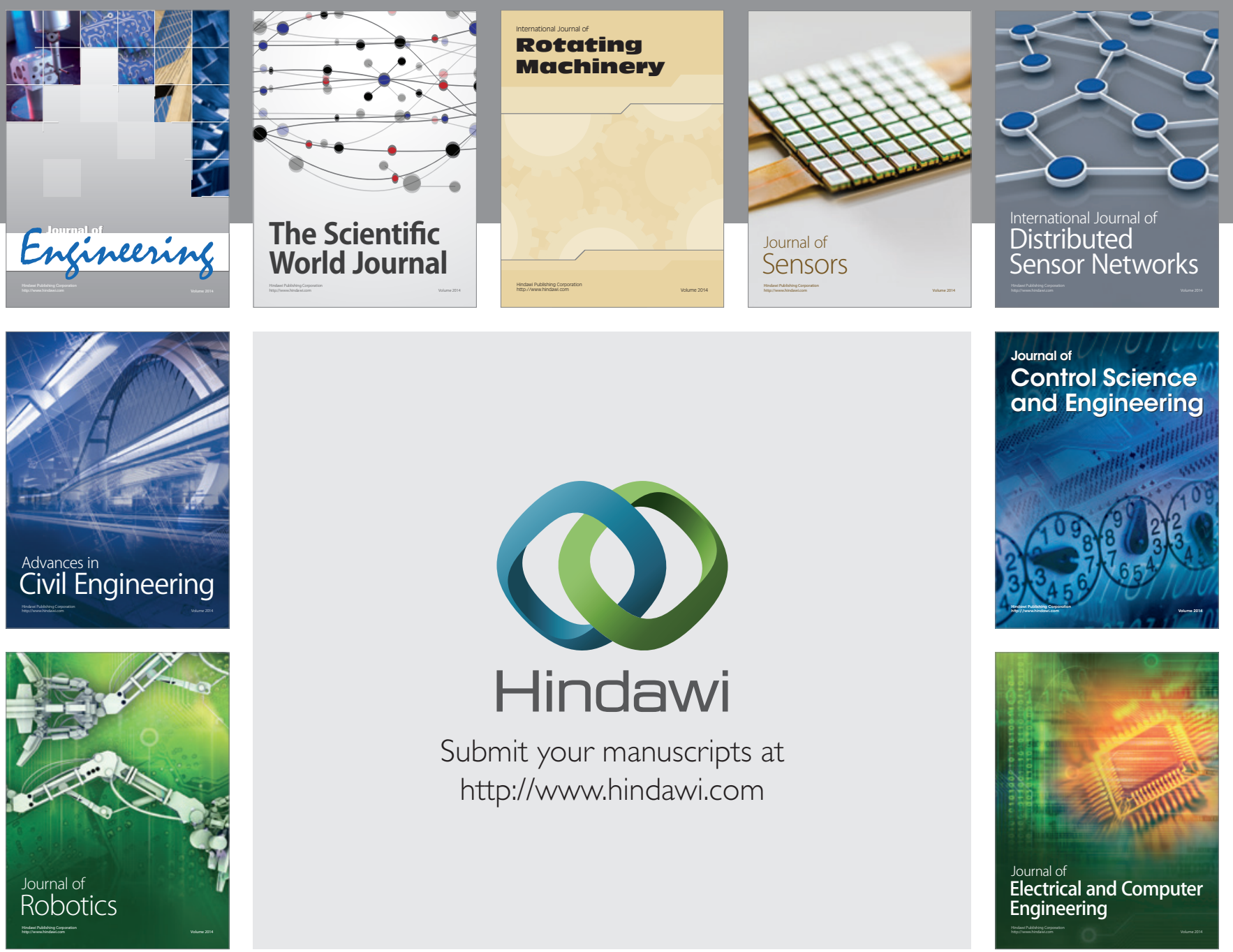

Submit your manuscripts at

http://www.hindawi.com
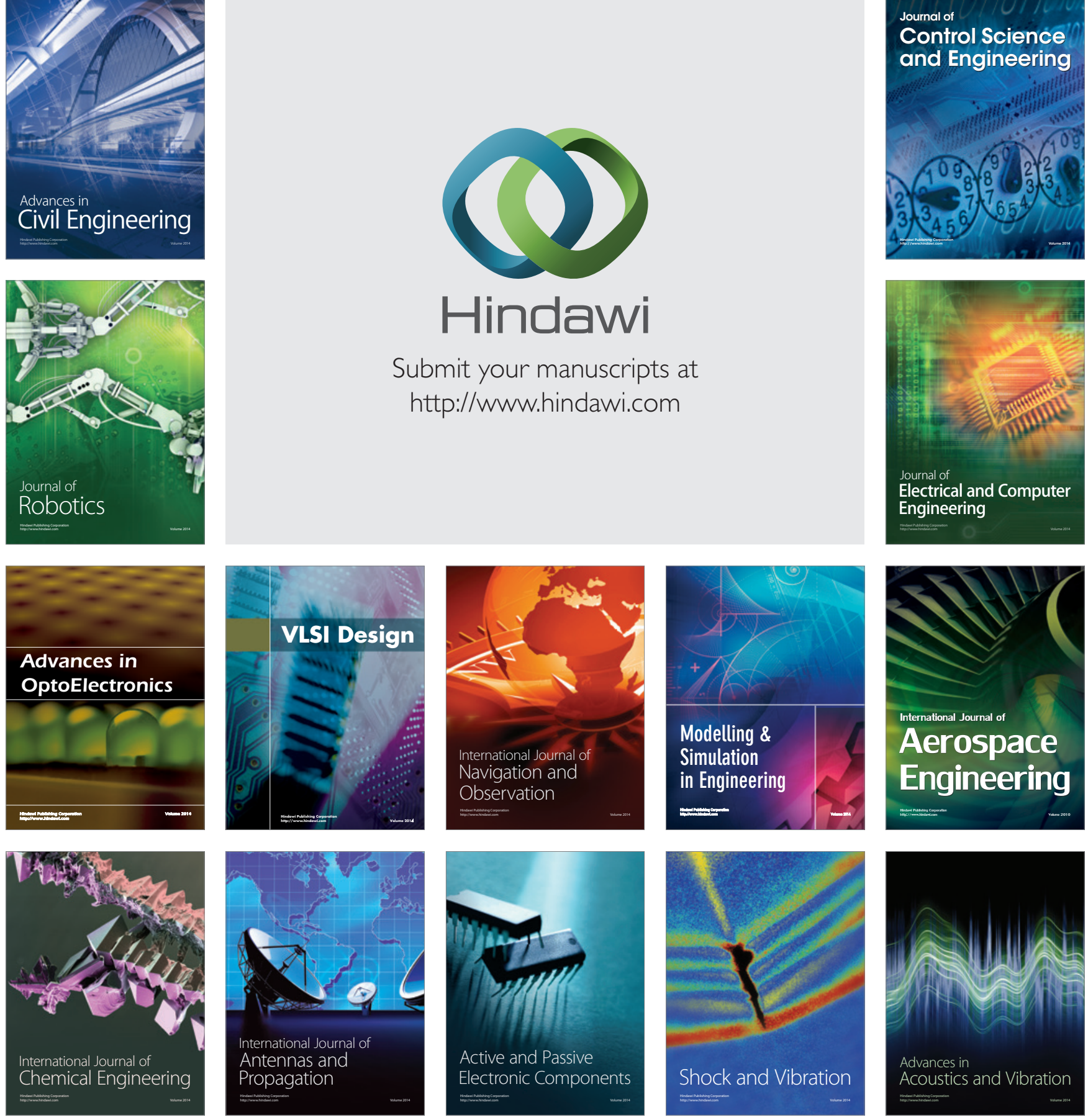